\title{
Measuring supermassive black holes with gas kinematics
}

\section{The LINERs IC 989, NGC 5077, and NGC 6500^}

\author{
G. De Francesco ${ }^{1}$, A. Capetti ${ }^{1}$, and A. Marconi ${ }^{2}$ \\ 1 INAF - Osservatorio Astronomico di Torino, Strada Osservatorio 20, 10025 Pino Torinese, Italy \\ e-mail: [defrancesco; capetti]@oato.inaf.it \\ 2 Dipartimento di Astronomia e Scienza dello Spazio, Università di Firenze, Largo E. Fermi 2, 50125 Firenze, Italy \\ e-mail: marconi@arcetri.astro.it
}

Received 29 August 2007 / Accepted 26 October 2007

ABSTRACT

\begin{abstract}
We present results from a kinematical study of the gas in the nucleus of a sample of three LINER galaxies, obtained from archival HST/STIS long-slit spectra. We found that, while for the elliptical galaxy NGC 5077, the observed velocity curves are consistent with gas in regular rotation around the galaxy's center, this is not the case for the two remaining objects. By modeling the surface brightness distribution and rotation curve from the emission lines in NGC 5077, we found that the observed kinematics of the circumnuclear gas can be accurately reproduced by adding to the stellar mass component a black hole mass of $M_{\mathrm{BH}}=6.8_{-2.8}^{+4.3} \times 10^{8} M_{\odot}$ (uncertainties at a $1 \sigma$ level); the radius of its sphere of influence $\left(R_{\mathrm{sph}} \sim 0^{\prime} \cdot 34\right)$ is well-resolved at the HST resolution. The BH mass estimate in NGC 5077 is in fairly good agreement with both the $M_{\mathrm{BH}}-M_{\text {bul }}$ (with an upward scatter of $\sim 0.4$ dex) and $M_{\mathrm{BH}}-\sigma$ correlations (with an upward scatter of $0.5 \mathrm{dex}$ in the Tremaine et al. form and essentially no scatter using the Ferrarese et al. form) and provides further support for the presence of a connection between the residuals from the $M_{\mathrm{BH}}-\sigma$ correlation and the bulge effective radius. This indicates the presence of a black hole's "fundamental plane" in the sense that a combination of at least $\sigma$ and $R_{\mathrm{e}}$ drives the correlations between $M_{\mathrm{BH}}$ and host bulge properties.
\end{abstract}

Key words. black hole physics - galaxies: active - galaxies: bulges - galaxies: nuclei - galaxies: kinematics and dynamics

\section{Introduction}

It is now clear that the presence of a supermassive black hole $(\mathrm{SMBH})$ is a common, if not universal, feature in the center of galaxies. In fact, since active galactic nuclei (AGNs) are thought to be powered by mass accretion onto an SMBH, the high incidence of low-luminosity AGN activity in nearby galaxies (Heckman 1980; Maoz et al. 1995; Ho et al. 1997a,b; Braatz et al. 1997; Barth et al. 1998, 1999; Nagar et al. 2002) has led to the conclusion that a significant fraction of galaxies in the Local Universe must host SMBH. This conclusion is now supported by direct measurements of SMBH masses in the centers of nearby galaxies obtained with different techniques (see Ferrarese \& Ford 2005, for a review). These measurements indicate that the $\mathrm{BH}$ mass $M_{\mathrm{BH}}$ is related to the properties of the host galaxy, such as bulge luminosity $L_{\text {bul }}$ and mass $M_{\text {bul }}$ (Kormendy \& Richstone 1995; Magorrian et al. 1998; Marconi \& Hunt 2003), light concentration (Graham et al. 2001; Graham $\&$ Driver 2007), and bulge velocity dispersion $\sigma_{\text {bul }}$ (Ferrarese \& Merritt 2000; Gebhardt et al. 2000; Tremaine et al. 2002).

The existence of any correlations between $M_{\mathrm{BH}}$ and host bulge properties supports the idea that the growth of SMBHs and the formation of bulges are closely linked (Silk \& Rees 1998; Haehnelt \& Kauffmann 2000). This has profound implications for the process of galaxy formation and evolution. Moreover, SMBH mass estimates inferred via the above correlations, when

\footnotetext{
* Based on observations obtained at the Space Telescope Science Institute, which is operated by the Association of Universities for Research in Astronomy, Incorporated, under NASA contract NAS 5-26555.
}

more direct methods are not feasible, enter into a variety of important studies spanning from AGNs physics to the coeval formation and evolution of the host galaxy and its nuclear black hole.

These results need, however, to be further investigated by increasing the number of accurate $\mathrm{BH}$ mass determinations in nearby galactic nuclei to set these correlations on a stronger statistical basis. In particular, such a study has the potential of establishing the precise role of the various host galaxy's parameters in setting the resulting BH mass. To date, reliable SMBH detections have been obtained for a limited number of galaxies ( 30, Ferrarese \& Ford 2005), with the bulk of $M_{\mathrm{BH}}$ estimates in the range of $10^{7}-10^{9} M_{\odot}$. To add reliable new points to the $M_{\mathrm{BH}}-$ host galaxy's properties planes is then a fundamental task for future developments of astronomical and physical studies.

One widely applicable and relatively simple method of detecting BHs is based on gas kinematics (e.g. Harms et al. 1994; Ferrarese et al. 1996; Macchetto et al. 1997; Barth et al. 2001), through studies of emission lines from circumnuclear gas disks, provided that the gas velocity field is not significantly influenced by non gravitational motions. However, the purely gravitational kinematics of the gas can be established a posteriori from successful modeling of the gas velocity field under the sole influence of the stellar and black hole potential.

The Space Telescope Imaging Spectrograph (STIS) onboard $H S T$ is still the most suitable instrument for such studies as it provides a high angular resolution $(\sim 0,1)$ in the optical spectral region. In this band brighter emission lines are found with respect to the infrared, the only band accessible at high resolution with ground-based adaptive optics telescopes. The wealth 
Table 1. Sample of galaxies and STIS data.

\begin{tabular}{lccccccc}
\hline \hline Galaxy & Type & $\begin{array}{c}D \\
(\mathrm{Mpc})\end{array}$ & $\begin{array}{c}\sigma_{\text {star }} \\
\left(\mathrm{km} \mathrm{s}^{-1}\right)\end{array}$ & $N_{\text {slit }}$ & PA & $\begin{array}{c}\text { Exp. } T . \\
(\mathrm{s})\end{array}$ & Date \\
\hline IC 989 & E & 102 & 185 & 3 & -157 & 494 & $1998-03-13$ \\
NGC 5077 & E3-4 & 38 & 256 & 3 & -81 & 418 & $1998-03-12$ \\
NGC 6500 & Sab & 42 & 200 & 5 & 15 & 236 & $1998-11-03$ \\
\hline
\end{tabular}

of unpublished data contained in STIS archives represents an extraordinary and still unexplored resource. With the aim of finding galaxy candidates to provide a successful SMBH mass measurement, we performed a systematic search for unpublished data in the HST STIS archive.

In this paper we present the results obtained for a sample of three LINER galaxies. The galaxies were observed with STIS on HST during Cycle 7 under Proposal ID 7354 and were part of a larger sample of eight selected objects. The results on NGC 3998 have already been published (De Francesco et al. 2006, hereafter Paper I). In a forthcoming paper, we will complete the proposal targets and discuss the relation between BH mass, host galaxy properties, and nuclear activity. Table 1 lists the galaxies and their main physical properties. Basic data are from the Lyon/Meudon Extragalactic Database (HyperLeda) or NASA/IPAC Extragalactic Database (NED). Distances are calculated with $H_{0}=75 \mathrm{~km} \mathrm{~s}^{-1} \mathrm{Mpc}^{-1}$ and are corrected for Local Group infall onto Virgo.

The paper is organized as follows. In Sect. 2 we present HST/STIS data and the reduction that lead to the results described in Sect. 3. In Sect. 4 we model the observed emissionline rotation curve for NGC 5077 and show that the dynamics of the circumnuclear gas can be accurately reproduced by circular motions in a thin disk when a point-like dark mass is added to the stellar potential. Our results are discussed in Sect. 5, and summarized in Sect. 6.

\section{HST data and reduction}

The three galaxies considered were observed with STIS on HST with the G750M grating and the $52^{\prime \prime} \times 0$.' 1 slit. Data were acquired at different slit positions, following a perpendicular-to-slit pattern with a step of 0 . $^{\prime} 1$ and the central slit centered on the nucleus. The spectra obtained for each source, NUC for the nuclear slit, N1-N (to North), and S1-S (to South) for the off-nuclears, were retrieved from the public archive. Table 1 lists the number of slit positions, the orientation of the slit (from north to east), the exposure time for each positioning, and the observation date for the galaxies of the sample.

The data were obtained with a $2 \times 1$ on-chip binning of the detector pixels and automatically processed through the standard CALSTIS pipeline to perform the steps of bias and dark subtraction, applying the flat field and combining the two sub-exposures to reject cosmic-ray events. The data were then wavelength and flux calibrated with conversion to heliocentric wavelengths and absolute flux units and rectified for the geometric distortions. The 2-D spectral image obtained for each slit position has a spatial scale of 0 .'$^{\prime} 0507$ pixel $^{-1}$ along the slit, a dispersion of $\Delta \lambda=1.108 \AA$ pixel $^{-1}$, and a spectral resolution of $\mathcal{R} \simeq 3000$, covering the rest frame wavelength range 6480-7050 .

For each spectrum we selected the regions containing the lines of interest. The lines were fitted, row by row, along the dispersion direction, together with a linear continuum, with Gaussian functions using the task SPECFIT in STSDAS/IRAF. All emission lines present in the spectra $(\mathrm{H} \alpha$,

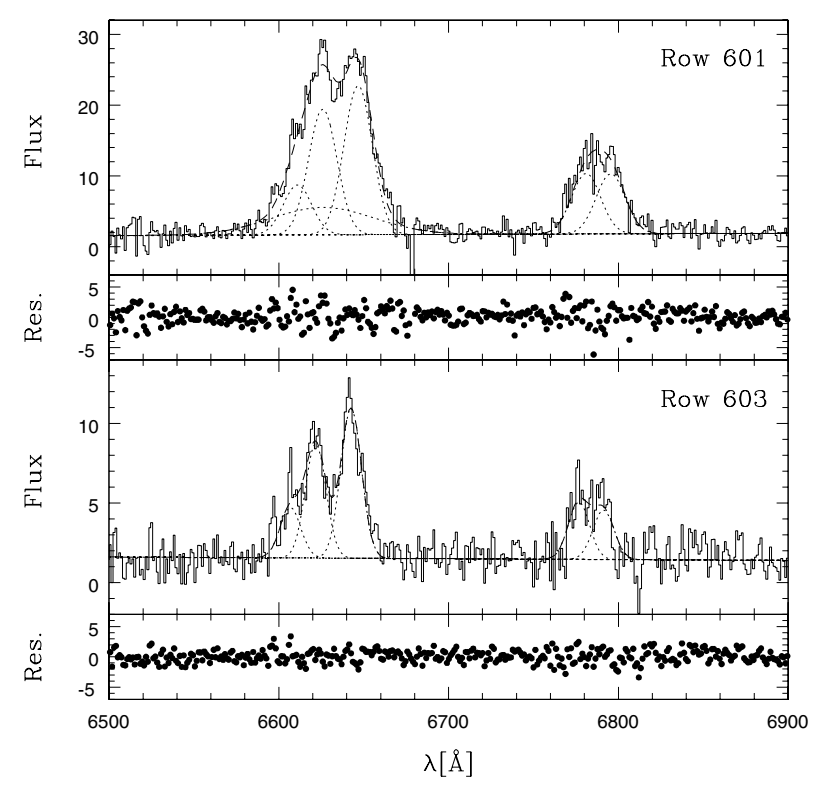

Fig. 1. Nuclear spectra of NGC 5077: at the slit center position (upper panels), showing the possible presence of a weak broad $\mathrm{H} \alpha$ component, and at 0.1 from it (lower panels). The best model spectrum (dashed line) is superposed on the data; dotted lines are for the Gaussians and linear continuum components of the fit. Flux is in units of $10^{-15} \mathrm{erg} \mathrm{cm}^{-2} \mathrm{~s}^{-1} \AA^{-1} \operatorname{arcsec}^{-2}$. The residuals from the fits are shown in the smaller panels.

[N II] $\lambda \lambda 6548,6583$ and [S II] $\lambda \lambda 6716,6731)$ were fitted simultaneously with the same velocity and width and with the relative flux of the [N II] lines kept fixed to 0.334 . The assumption relative to the sharing of the same kinematics for the emission lines will result in average kinematical quantities, weighted with the line surface brightness ${ }^{1}$.

In the regions where the signal-to-noise ratio (SNR) was insufficient, the fitting was improved by co-adding two or more pixels along the slit direction. As an example of the quality of data, Fig. 1 shows the spectrum of NGC 5077, the only galaxy of the group with a marginal detection of a broad $\mathrm{H} \alpha$ component, at two different positions along NUC slit.

\section{Results}

The results obtained from the fitting procedure for the three galaxies are shown in Figs. 2 through 4, where we plot the kinematical quantities, central velocity, and velocity dispersion, together with the surface brightness of the emission line with the best SNR at each location along the slit. The position-velocity diagram for the off-nuclear with the best quality of data is also reproduced in the upper panels of Figs. 2 and 4 for two sources of the program. In the following we discuss the results obtained for each galaxy of the sample.

\footnotetext{
${ }^{1}$ However, we investigated the possibility that lines did not share common kinematics, but we did not find evidence of this.
} 


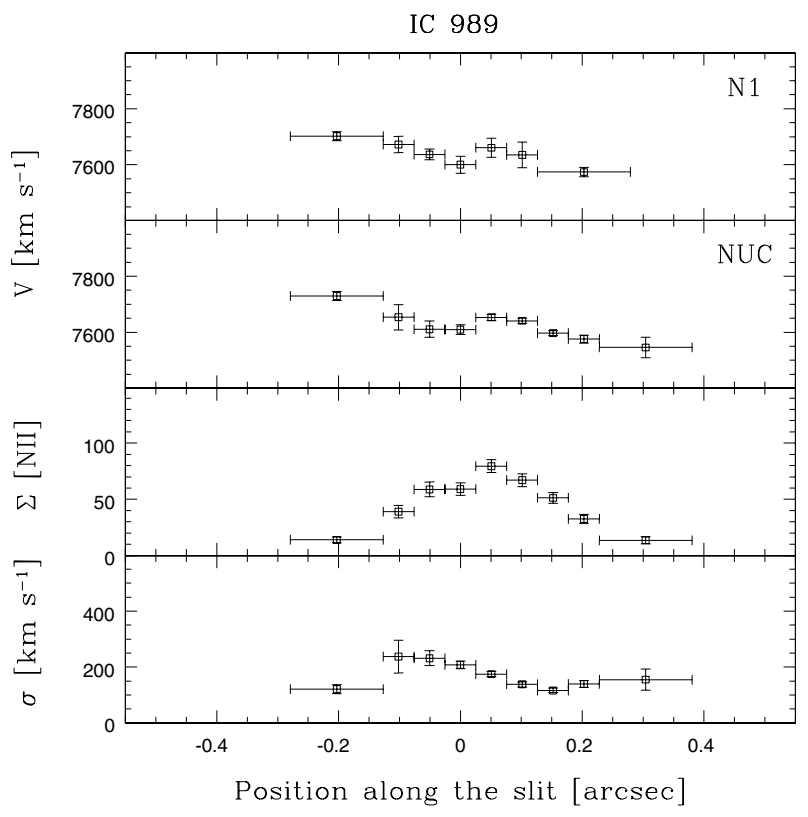

Fig. 2. Upper panel: velocity curve from the off-nuclear slit with the best quality of data. Lower panels: velocity, surface brightness, and velocity dispersion along NUC slit. Surface brightness is in units of $10^{-15} \mathrm{erg} \mathrm{s}^{-1} \mathrm{~cm}^{-2} \operatorname{arcsec}^{-2}$. Positions along the slit are relative to the continuum peak.

\subsection{IC 989}

The velocity curve on the nuclear slit shows a large-scale rotation with redshift at the negative space values (NE region) and blueshift at the positive (SW region) (Fig. 2). The same trend is observable in the off-nuclear slits, with almost the same values of velocity gradients. However, the velocity gradient is reversed at the very center, suggesting the presence of a counter-rotating nuclear gas disk.

\subsection{NGC 5077}

For the elliptical galaxy NGC 5077, the observed velocity curves are apparently consistent with gas in regular rotation around the galaxy's center. The velocity curve in the central slit, NUC (Fig. 3, central panel), has a full amplitude of $\sim 400 \mathrm{~km} \mathrm{~s}^{-1}$ and shows a general reflection symmetry: starting from the center, the velocity rises rapidly on both sides by $\sim 200 \mathrm{~km} \mathrm{~s}^{-1}$ reaching a peak at $r \sim 0$.' 1 from the center. This trend is followed by a small decrease and a substantially constant value at larger radii. Line emission and velocity dispersion are strongly peaked and smoothly decrease from the nucleus outwards.

The behavior seen in the off-nuclear slits is qualitatively similar to what is seen at the NUC location (Fig. 3, left and right panels), but with smaller velocity amplitude and, more important, a less extreme velocity gradient. Both the amplitude and gradient decrease at increasing distance of the slit center from the nucleus, with a behavior characteristic of gas rotating in a circumnuclear disk.

Ground-based photometric and kinematical studies (Demoulin-Ulrich et al. 1984; Bertola et al. 1991) show that NGC 5077 exhibits a gaseous disk with the major axis roughly orthogonal to the galaxy photometric major axis $\left(\mathrm{PA} \sim 10^{\circ}\right)$. The gas isophotes show twisting and a marked warp on the W side at $r \sim 20^{\prime \prime}$ (Caon et al. 2000). The gas has a fairly symmetric and smooth rotation curve at PA $=108^{\circ}(\sim$ major axis of the ionized gas distribution) with a half amplitude of $\sim 270 \mathrm{~km} \mathrm{~s}^{-1}$ at $r \sim 13^{\prime \prime}$. At PA $=10^{\circ}$, the stellar rotation curve exhibits a counter-rotating core $\left(r \leq 5^{\prime \prime}\right)$. Along this axis, the gas rotates in the same direction as the stellar nucleus and shows a small-scale central velocity plateau.

\subsection{NGC 6500}

The velocity curves for this galaxy are complex (see Fig. 4). On the nuclear slit, the velocity apparently shows a general rotational trend, with redshift on the left and blueshift on the right side of the diagram. The same large-scale rotation is observable on the off-nuclear slit positions. Smaller scale high-amplitude $\left(\sim 200 \mathrm{~km} \mathrm{~s}^{-1}\right)$ velocity oscillations are also clearly visible.

In the SW region at $r \leq 00^{\prime} 4$ the position-velocity diagrams of NUC and the adjacent S1 slit show a trend that is inconsistent with gas in regular rotation around the galaxy's center (see Fig. 4, second upper panel). In this region the velocity curves diverge, reaching a separation of $\sim 300 \mathrm{~km} \mathrm{~s}^{-1}$ at $r \sim 0$.' 2 . The over-plot of the position velocity diagrams suggests an expanding bubble of gas.

Summarizing, we find that NGC 5077 shows a line emission consistent with a circumnuclear gas disk in Keplerian rotation around the galaxy's center. For the remaining two objects, the trend of their velocity curves cannot be ascribed to the regular rotation of a nuclear gas disk. The position-velocity diagrams of IC 989 indicate the presence of a counter-rotating gas component. Modeling this configuration would require (at least) a warped geometry for the disk, which cannot be constrained with the present data. By these considerations, only NGC 5077 is a suitable candidate for providing a successful SMBH mass measurement.

\section{Modeling the rotation curves of NGC 5077}

Our modeling code, described in detail in Marconi et al. (2003), was used to fit the observed rotation curves of NGC 5077. The code computes the rotation curves of the gas assuming that the gas is rotating in circular orbits within a thin disk in the galaxy potential. The gravitational potential has two components: the stellar potential (whose mass distribution will be determined in Sect. 4.1), characterized by its mass-to-light ratio and a dark mass concentration (the black hole), spatially unresolved at HST+STIS resolution and characterized by its total mass $M_{\mathrm{BH}}$. In computing the rotation curves, we take into account the finite spatial resolution of the observations, and the intrinsic surface brightness distribution (ISBD) of the emission lines and we integrate over the slit and pixel area. The adopted HST+STIS point spread function (PSF) is obtained by fitting with three Gaussians the Tiny Tim (Krist \& Hook 1999) model PSF calculated at $6700 \AA$ without the Lyot-stop, according to the procedure suggested by Dressel (2006). The $\chi^{2}$ is minimized to determine the free parameters using the downhill simplex algorithm by Press et al. (1992).

\subsection{The stellar mass distribution}

To assess the contribution of stars to the gravitational potential in the nuclear region, we derived the stellar luminosity density from the observed surface brightness distribution. We reconstructed the galaxy light profile using a WFPC2 F547M ( $V$ band) image retrieved from the public archive (Fig. 5). Based on WFPC2 F702W ( $R$ band) images, Tran et al. (2001) found a filamentary 

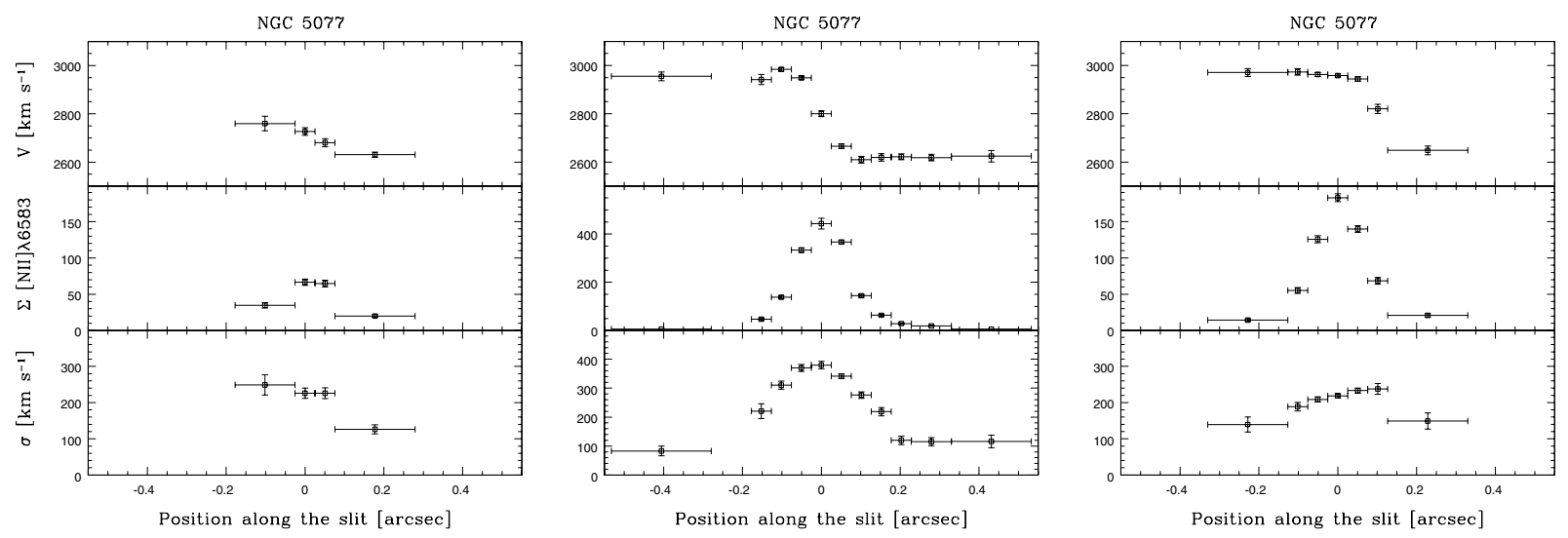

Fig. 3. Velocity, surface brightness, and velocity dispersion along the off-nuclear slit position N1 (left panel), NUC (central panel), and S1 (right). Positions along the slits are relative to the continuum peak, positive values are NW.

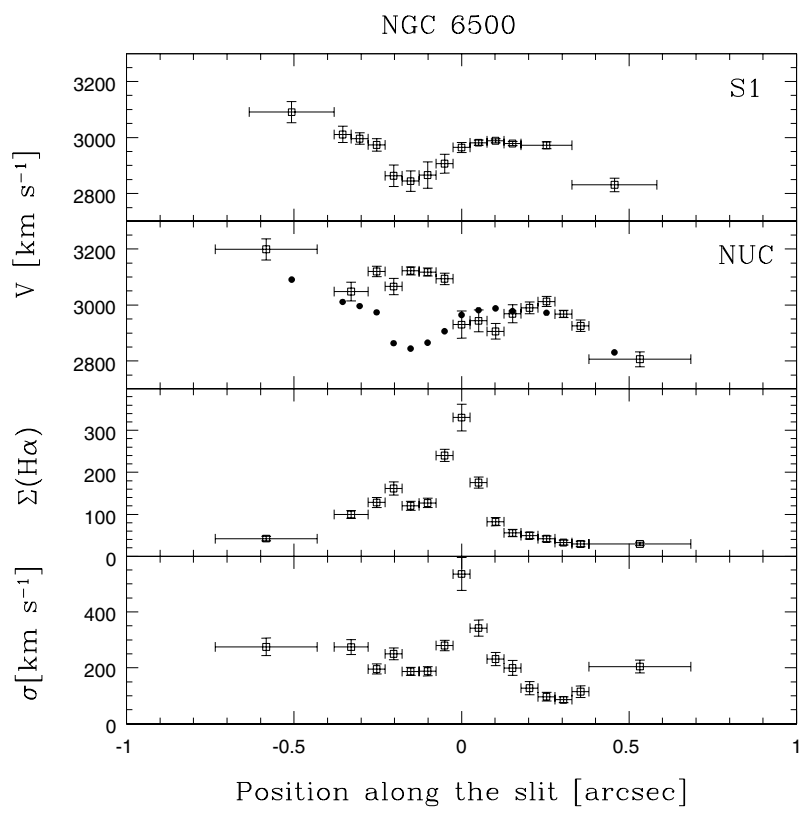

Fig. 4. Same as Fig. 2. Second upper panel: over-plot of NUC (empty squares) and $\mathrm{S} 1$ (filled circles) velocity curves showing the inconsistency of the gas position-velocity diagram with regular rotation of a nuclear gas disk.

dust structure in NGC 5077, with position angle of the major axis of the main dust feature $\sim 102^{\circ}$ (at $r=10^{\prime \prime}$ ). Our inspection of $H S T$ archive images, however, shows that the influence of dust is negligible. In fact, Tran et al. (2001) derived a mean visual extinction of only $\left\langle A_{V}\right\rangle=0.048$. We used the IRAF/STSDAS program ELLIPSE to fit elliptical isophotes to the galaxy (see Fig. 6). Nuclear regions ( $r \leq 0.3$ ) show the largest ellipticity variations. At larger radii, ellipticity and position angle show small variations around the values 0.25 and $10^{\circ}$, respectively.

We derived the stars' density profile from the galaxy surface brightness following the same method described in Paper I when assuming an oblate spheroid density distribution. Following van der Marel \& van den Bosch (1998), the stellar density distribution was parameterized as

$\rho(m)=\rho_{0}\left(\frac{m}{r_{\mathrm{b}}}\right)^{-\alpha}\left[1+\left(\frac{m}{r_{\mathrm{b}}}\right)^{2}\right]^{-\beta}$ where $m$ is given by $m^{2}=x^{2}+y^{2}+z^{2} / q^{2}$, xyz is a reference system with the $x y$ plane corresponding to the principal plane of the potential and $q$ is the intrinsic axial ratio. The above stellar density is integrated along the line of sight, to provide the surface brightness distribution on the plane of the sky, and is then convolved with the telescope+instrument PSF (derived from Tiny Tim) and averaged over the detector pixel size to obtain the observed surface brightness distribution (for details see Marconi et al. 2003). The de-projection was performed by adopting the reference value of $\Upsilon_{V}=1$ for the mass-to-light ratio in the $V$ band (its real value will be determined in Sect. 4.2). As the intrinsic potential axial ratio $q$ is related to the inclination of the principal plane with respect to the line of sight by the observed isophote ellipticity, we are left with the freedom of assuming different galaxy inclinations to the line of sight. We performed the de-projection adopting the value $i=44^{\circ}$ for the galaxy inclination, as given from the HyperLeda database. Due to the small ellipticity of this galaxy, the precise value of its inclination only has a marginal effect on the resulting mass distribution. The best fit obtained is shown in Fig. 7 with $\alpha=0.47, \beta=0.79$, and $r_{\mathrm{b}}=0.59^{\prime \prime}$.

\subsection{Fitting the gas kinematics}

The observed kinematical quantities are averages over apertures defined by the slit width and the detector pixel size along the slit. The model fitting to the observed kinematical quantities thus depends on the intrinsic emission-line surface-brightness distribution, which is the weight for the averaging process, and on the following parameters:

- the black hole mass $M_{\mathrm{BH}}$;

- the mass-to-light ratio of the stellar component, $\Upsilon_{V}$;

- the position of the kinematical center in the plane of the sky, $x_{0}, y_{0}$, with the reference frame centered on the nuclear slit and $x$ axis aligned with the slit;

- the inclination of the rotating disk, $i$;

- the angle between the slits and the line of nodes, $\theta$;

- the systemic velocity of the disk, $v_{\text {sys. }}$.

With respect to Paper I, the modeling code has been slightly improved, so it now allows us to 1) fit the surface brightness and kinematical data simultaneously and 2) set limits to the parameters range. We used the second feature in particular to constrain the mass-to-light ratio to $\Upsilon_{V}<7.87$, as derived from Maraston (1998) for a Salpeter initial mass function (IMF). 

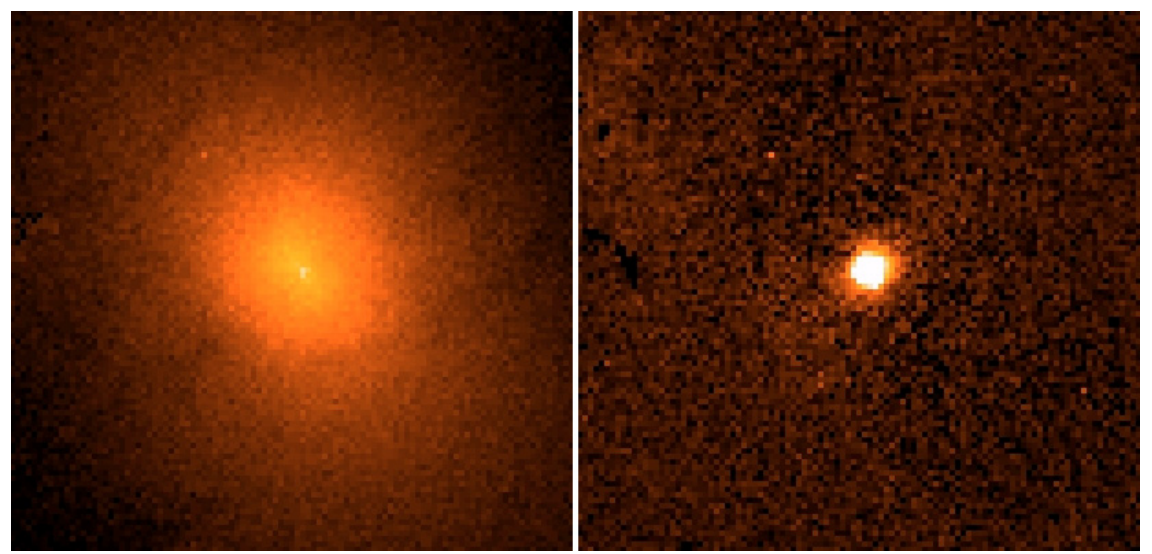

Fig. 5. Left: $V$ band (F547M filter) image of NGC 5077. Right: H $\alpha$ WFPC2/HST image. Field of view 4". $5 \times 4$ "' 5 . North is up, East is left.

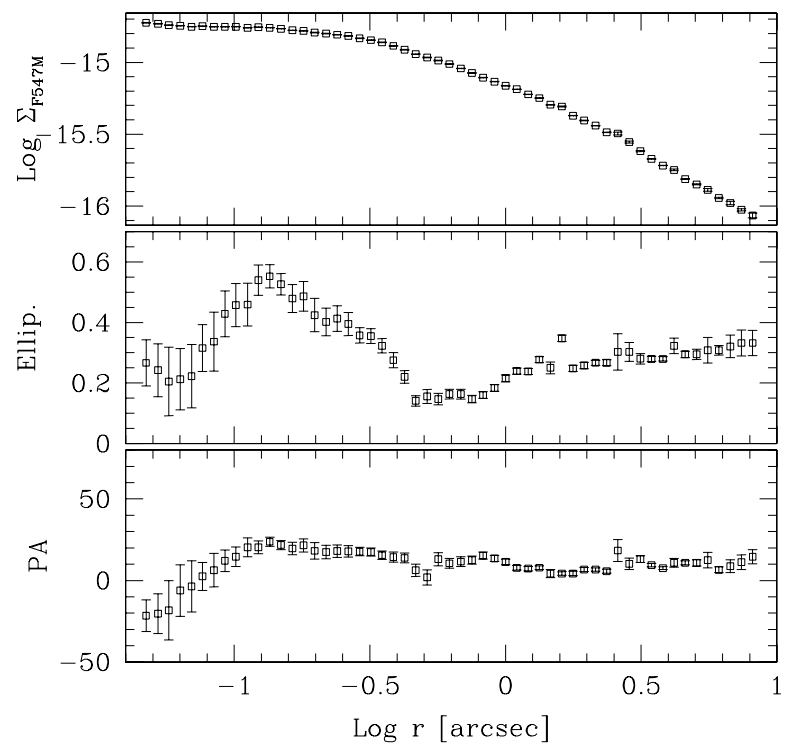

Fig. 6. Results of the isophotes analysis of the $\mathrm{V}$ band image of NGC 5077. Surface brightness is shown in the top panel (in units of $\operatorname{erg~s}^{-1} \mathrm{~cm}^{-2} \AA^{-1} \operatorname{arcsec}^{-2}$ ), and the galaxy's ellipticity and position angle are shown in the middle and bottom panels, respectively.

A crucial issue for modeling the gas kinematics is the inclination of the nuclear gas disk, as this is strongly coupled with the black hole mass and $\Upsilon_{V}$. In an oblate spheroid, the stable orbits of the gas are coplanar with the principal plane of the potential, and it is possible to directly associate the galaxy inclination and line of nodes with those of the circumnuclear gas. However, the potential shape is not determined well enough by the isophotal fitting down to the innermost regions of the galaxy, and it is possible that a change of principal plane might occur at the smallest radii, in particular within the sphere of influence of a supermassive black hole. We then performed a $\chi^{2}$ minimization for different values of $i$, namely $i=20^{\circ}, \ldots, 70^{\circ}$, allowing all other parameters to vary freely, including those describing the intrinsic line surface brightness distribution. Due to the sensitivity of the velocity dispersions to the ISBD modeling and to other computational problems (i.e. a coarse sampling), as shown by Marconi et al. (2006), we decided to initially restrict the kinematical fitting only to the velocity curves. We show in Sect. 4.2.1 that including the velocity dispersions in the fitting procedure only has a marginal effect on our results.

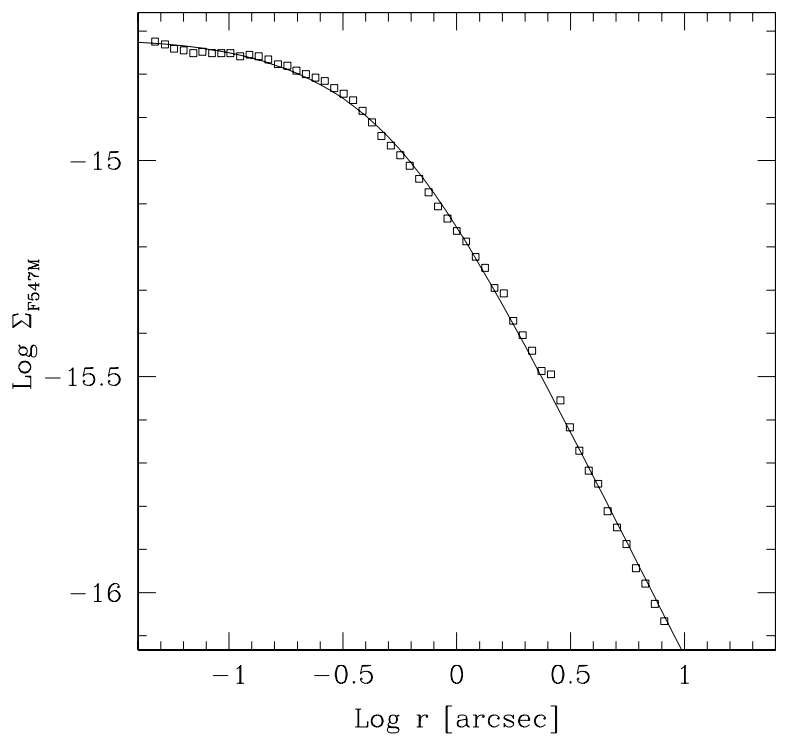

Fig. 7. Fit to the surface brightness profile obtained from an oblate spheroid stellar density distribution.

Inspection of Fig. 3 suggests that the ISBD should be described at least by 2 components: one at the NUC slit (the nuclear component), plus a more extended one (base component). The intrinsic emission line surface brightness was modeled with a composition of two circularly symmetric Gaussians and a constant function (having as free parameters the amplitudes, scale radii, and the positions on the sky plane of the symmetry centers). Marconi et al. (2006) have recently shown that the choice of a particular model for the line surface brightness distribution affects the final $\mathrm{BH}$ mass estimate very little, provided that the model reproduces the observed line emission within the errors. They showed that systematic errors on $M_{\mathrm{BH}}$ due to the adopted intrinsic emission line surface brightness were on the order of 0.05 in $\log M_{\mathrm{BH}}$ for their STIS data. We also tested the effect, for our data, of using exponential functions in the ISBD modeling. The BH masses derived were $\lesssim 0.03$ in $\log M_{\mathrm{BH}}$ lower than obtained using a Gaussian ISBD.

From the best fits obtained at varying gas disk inclination, we quoted the goodness of the fits only with respect to the velocity curves, since we are interested in the kinematical model that reproduces the position-velocity data best. Excluding the contribution to the $\chi^{2}$ of the ISBD fit is a conservative choice for our data, as we verified that quoting the global fits would 

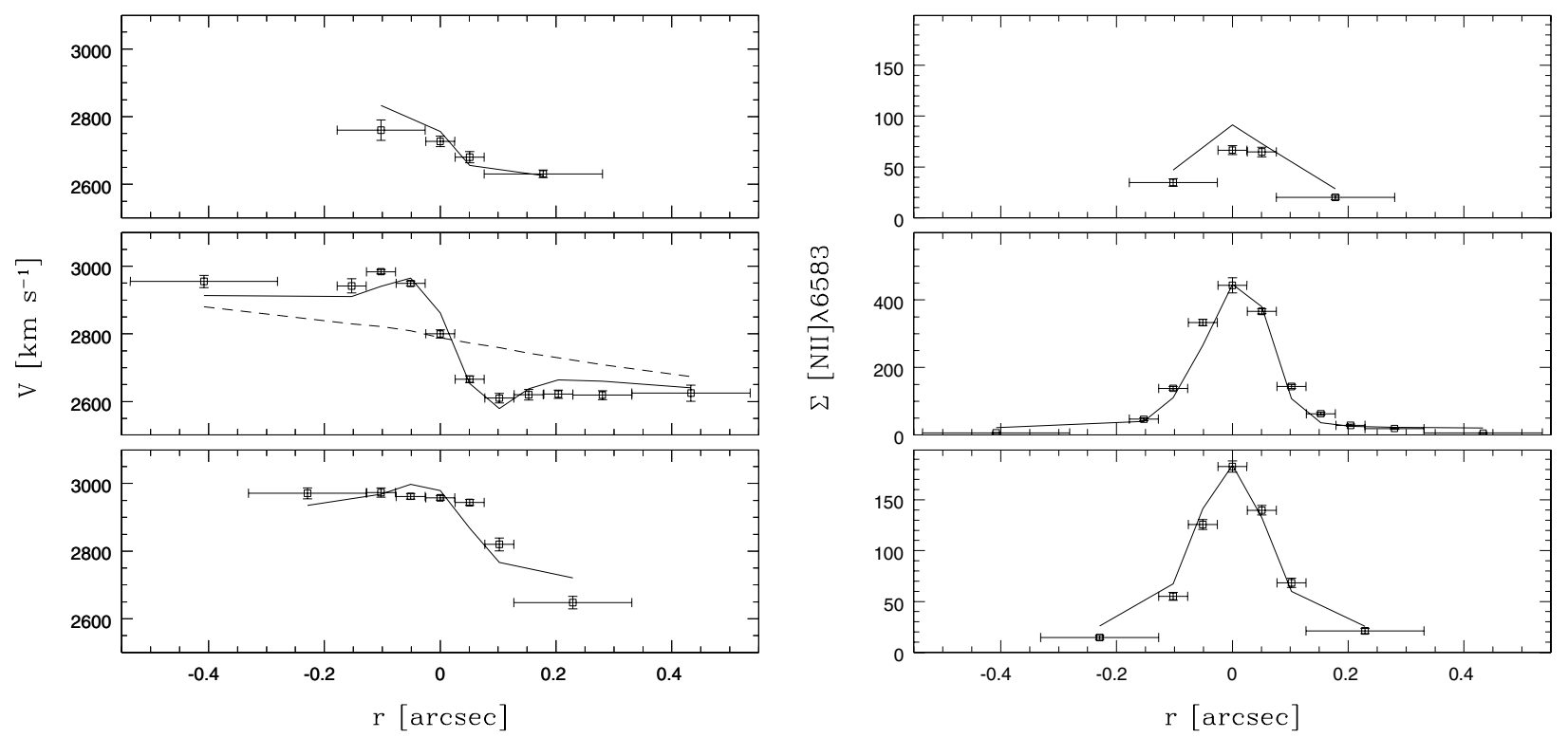

Fig. 8. Overall best fit to the rotation curves (left) with the case of null $\mathrm{BH}$ mass (dashed line) superposed on the central slit. Corresponding fit to the line surface brightness distribution (right). From upper to bottom panel: N1, NUC, S1.

Table 2. Overall best-fit parameters.

\begin{tabular}{|c|c|c|c|c|c|c|c|}
\hline $\begin{array}{c}i \\
\left(^{\circ}\right)\end{array}$ & $\begin{array}{c}x_{0} \\
\left({ }^{\prime \prime}\right)\end{array}$ & $\begin{array}{c}y_{0} \\
\left({ }^{\prime \prime}\right)\end{array}$ & $\begin{array}{c}\theta \\
\left(^{\circ}\right)\end{array}$ & $\begin{array}{c}v_{s y s} \\
\left(\mathrm{~km} \mathrm{~s}^{-1}\right)\end{array}$ & $\begin{array}{c}\Upsilon_{V} \\
\left(M_{\odot} / L_{\odot}\right)_{V}\end{array}$ & $\begin{array}{l}M_{\mathrm{BH}} \\
\left(M_{\odot}\right)\end{array}$ & $\chi_{r}^{2}$ \\
\hline 40 & 0.03 & -0.01 & 34 & 2780 & 7.7 & $6.8 \times 10^{8}$ & 15.49 \\
\hline
\end{tabular}

narrow the ranges of acceptable model parameters. The overall best-fitting model, presented in Fig. 8, is obtained for $i=40^{\circ}$, $M_{\mathrm{BH}}=6.8 \times 10^{8} M_{\odot}, \Upsilon_{V}=7.7$, and an angle from the slits to the line of nodes of $34^{\circ}$ (i.e. offset by $25^{\circ}$ from the groundbased measurements). Table 2 shows the best kinematical model parameters, together with the corresponding $\chi^{2}$ reduced value $\left(\chi_{r}^{2}=\chi^{2} /\right.$ d.o.f. with d.o.f. $=16$ degrees of freedom). Apparently, the best fit slightly underpredicts the large-scale velocity points. We then tested the effect of allowing a higher maximum value of the mass-to-light ratio, related to a different choice of the IMF. A fit was performed by adopting the value 13.78 as limit to $\Upsilon_{V}$, corresponding to the oldest stellar population and a giantdominated IMF. Indeed, the best fit obtained (with $\Upsilon_{V}=13.2$ ) reproduces the observed velocities better at large radii. However, the $\mathrm{BH}$ mass does not change significantly, resulting in $\log M_{\mathrm{BH}}$ only 0.02 lower than our previous result. We therefore preferred to maintain the constraint on $\Upsilon_{V}$ derived by adopting the standard Salpeter IMF.

The value of minimum $\chi_{r}^{2}$ (see Table 2) is far higher than the value indicative of a good fit. To establish the statistical significance of $\chi_{r}^{2}$ variations among different models, we followed Barth et al. (2001) and rescaled the error bars in our velocity measurements by adding a constant error in quadrature such that the overall best-fitting model provides $\chi_{r}^{2} \sim 1$. This is quite a conservative approach as it has the effect of increasing the final uncertainty on $M_{\mathrm{BH}}$. The additional velocity error is $45 \mathrm{~km} \mathrm{~s}^{-1}$. The rescaled $\chi_{r}^{2}$ values against inclination are reported in the bottom panel ${ }^{2}$ of Fig. 9. The acceptable models (within the $1 \sigma$

2 The different trend (high increase) of $\chi_{r}^{2}[$ scaled $]$ at small angles with respect to NGC 3998 (Paper I, Fig. 11) is determined by the a priori fixing of an upper limit for $\Upsilon_{V}$ values. confidence level, i.e. $\Delta \chi_{r}^{2} \leq 0.06$ for 16 d.o.f.), correspond to the range of inclination of $35^{\circ} \leq i \leq 50^{\circ}$.

The case of no black hole mass is also shown in Fig. 8 for the central slit. This model (obtained by constraining $\Upsilon_{V}<7.87$ and $v_{\text {sys }}$ to the same value of the best fit) predicts a velocity gradient that is too shallow with respect to the data, particularly in the central region $r \leq 0$ '. 2 , with a correspondingly unacceptable value of $\chi_{r}^{2}=7.9$ (properly rescaled to the overall best fit).

To evaluate the uncertainty associated to the black hole mass estimate, we explored its variation with respect to the parameter that is more strongly coupled to it, i.e. the mass-to-light ratio $\Upsilon_{V}$ (having already considered the dependence on orientation). The uncertainty on $M_{\mathrm{BH}}$ associated to changes in $\Upsilon_{V}$ has been estimated by building a $\chi_{r}^{2}$ grid in the $M_{\mathrm{BH}}$ vs. $\Upsilon_{V}$ parameter space. At each point of the grid, described by a fixed pair of $M_{\mathrm{BH}}$ and $\Upsilon_{V}$ values, we obtained the best fit model allowing all other parameters to vary freely and derived the corresponding $\chi_{r}^{2}$ value (properly rescaled). The result of this analysis at $i=40^{\circ}$ is presented in Fig. 10. The $1 \sigma$ range of the black hole mass at this disk inclination is $M_{\mathrm{BH}}=6.8_{-1.3}^{+2.1} \times 10^{8} M_{\odot}$, reported in the error bar in Fig. 9.

If we conservatively adopt the same fractional uncertainties on $M_{\mathrm{BH}}$, associated to variations in $\Upsilon_{V}$, for the allowed disk inclinations, we obtain a global range of acceptable black hole mass of $M_{\mathrm{BH}}=(4.0-11.1) \times 10^{8} M_{\odot}$ at a $1 \sigma$ level.

\subsubsection{Velocity dispersion distribution}

At this point in our analysis we tested the influence on the above results of including the velocity dispersions in the fitting procedure. The observed ionised-gas velocity dispersions are fairly large in NGC 5077, reaching almost $400 \mathrm{~km} \mathrm{~s}^{-1}$ in the nuclear slit. Velocity dispersions larger than expected from unresolved rotation could be an indication of non circular motions that could invalidate the $\mathrm{BH}$ mass estimate (Barth et al. 2001; Verdoes Kleijn et al. 2000, 2002; Cappellari et al. 2002). However, in the case of NGC 5077, rotational and instrumental broadening is sufficient to reproduce the behaviour of velocity dispersions. 


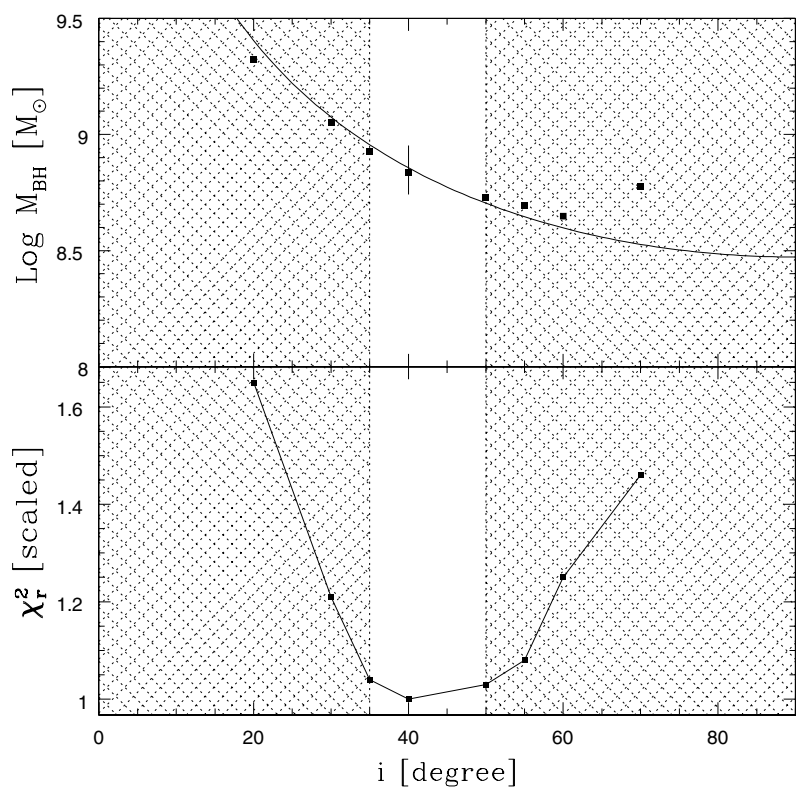

Fig. 9. Upper panel: best fit values of $M_{\mathrm{BH}}$ obtained by varying the disk inclination. The continuous line reproduces the $\propto 1 / \sin ^{2} i$ dependence of $M_{\mathrm{BH}}$. Lower panel: corresponding $\chi_{r}^{2}$ values scaled to the overall best fit. Inclinations smaller than $35^{\circ}$ or larger than $50^{\circ}$ are excluded at a confidence level of $1 \sigma$ (shaded regions in the diagrams), evaluated for 16 degrees of freedom.

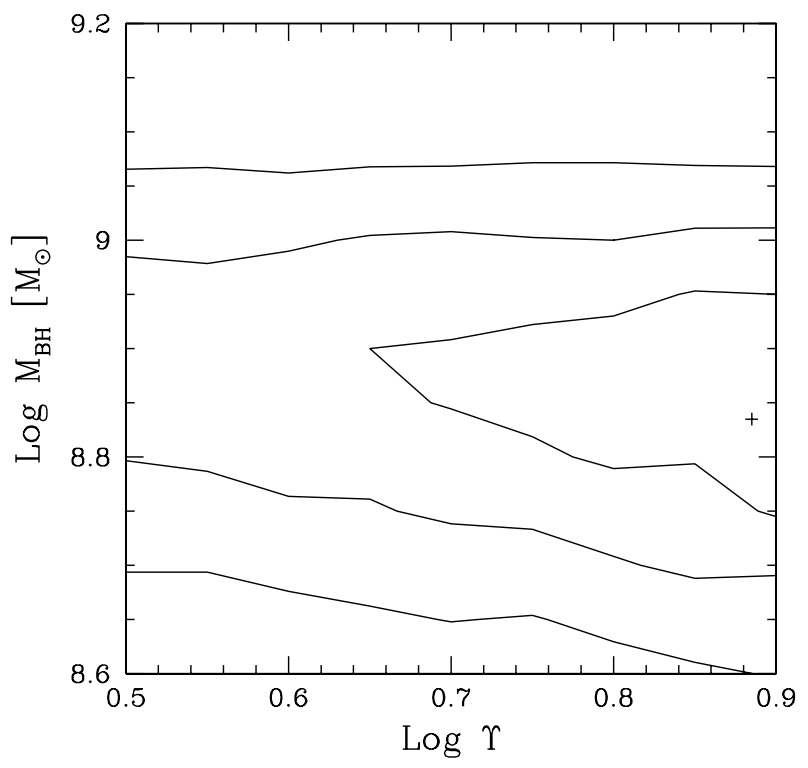

Fig. 10. $\chi_{r}^{2}$ contours at varying $\Upsilon_{V}$ and $M_{\mathrm{BH}}$ at an inclination of $i=40^{\circ}$. Contours are plotted for confidence levels of 1,2 , and $3 \sigma$. The plus sign marks the overall best fit.

First of all, by adopting the best fit parameter set (Table 2), we obtained the velocity dispersion distribution shown in Fig. 11. The observed velocity dispersions are reproduced acceptably well by the model, with only a small mismatch of observed and model peak positions. Thus the nuclear rise of the velocity dispersion is accounted for as unresolved rotation by the fitting model.

Furthermore, we repeated the $\chi^{2}$ minimization at $i=40^{\circ}$, this time by including the observed velocity dispersions in the fitting procedure (with no free parameters added). The best fit obtained is shown in Fig. 11. An improvement of the match with observed values is obtained, while there is a slight worsening

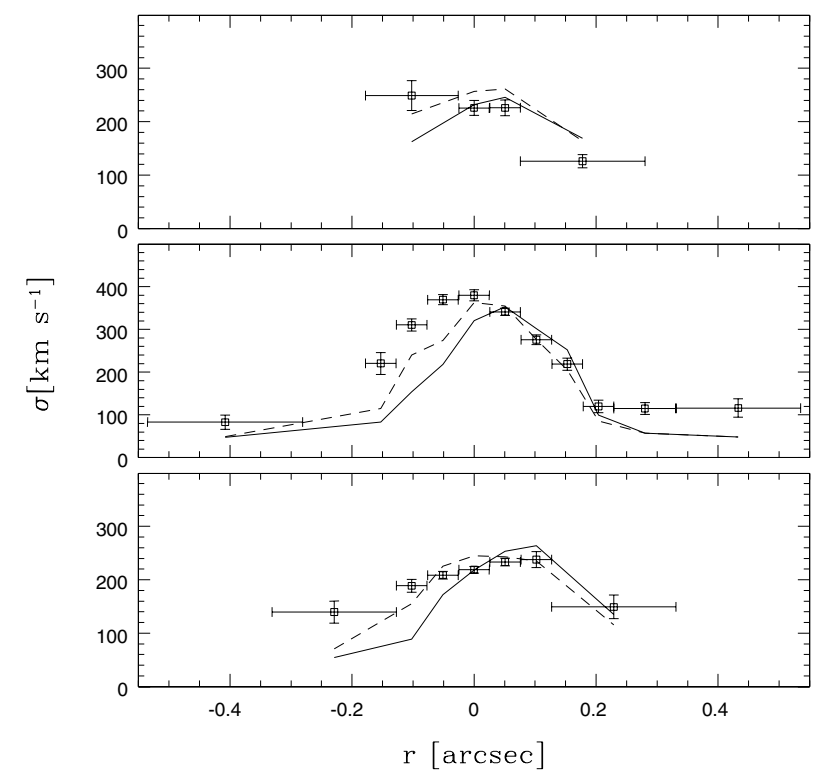

Fig. 11. Velocity dispersion distribution expected from the overall best fit model (solid line) at $i=40^{\circ}$, and derived (dashed line) by including the observed velocity dispersions in the fitting procedure. From upper to bottom panel: N1, NUC, S1.

of the fit to the velocity curves, with $\chi_{r}^{2}$ (scaled to the overall best fit) $=1.08$. The effect of including the line widths in the analysis results in increasing the $\mathrm{BH}$ mass by only $\sim 24 \%$ to $M_{\mathrm{BH}}=8.4 \times 10^{8} M_{\odot}$.

\section{Discussion}

The model fitting performed for NGC 5077 indicates that the kinematics of gas in its innermost regions is described correctly as circular motions in a thin disk when a point-like dark mass of $M_{\mathrm{BH}}=6.8_{-2.8}^{+4.3} \times 10^{8} M_{\odot}$ is added to the galaxy potential. We can now explore how BH mass relates to the properties of the host galaxy, including spheroid (bulge) mass (Marconi \& Hunt 2003), stellar velocity dispersion (Tremaine et al. 2002; Ferrarese \& Ford 2005), and light concentration (Graham \& Driver 2007).

Following Marconi \& Hunt (2003), we used the virial mass $M_{\text {vir }}=k R_{\mathrm{e}} \sigma_{\text {bul }}^{2} / G$, with $k=5$ (Cappellari et al. 2006) to determine the bulge mass of NGC 5077. We found discrepant values for the effective radius of the bulge, $R_{\mathrm{e}}$, in the literature. The values were derived through different methods (i.e. isophotal fitting, photometric aperture growth curves) and are 15'. 4 (Bender et al. 1989), 15".9 (Sánchez-Portal et al. 2004), 17'.3 (Poulain \& Nieto 1994), 19" (Bertola et al. 1991), 22"' 1 (Trujillo et al. 2004), 22". 8 (de Vaucouleurs et al. 1991, RC3), and 25" (Burstein et al. 1987). We decided to adopt the average of the above values, $R_{\mathrm{e}}=19^{\prime \prime} 6 \pm 3 . .7$ as the effective bulge radius of NGC 5077 . At the adopted distance of the galaxy, this value corresponds to $3.6 \pm 0.7 \mathrm{kpc}$.

The stellar velocity dispersion determinations we found in the literature are $285 \pm 28 \mathrm{~km} \mathrm{~s}^{-1}$ (Davies et al. 1987, $1^{\prime \prime} .5 \times 4^{\prime \prime}$ aperture), $252 \pm 12 \mathrm{~km} \mathrm{~s}^{-1}$ (Carollo et al. 1993, $1^{\prime \prime} .3 \times 5^{\prime \prime}$ and $1^{\prime \prime} .6 \times 5^{\prime \prime}$ apertures), and $263 \pm 5 \mathrm{~km} \mathrm{~s}^{-1}$ (Caon et al. 2000, $1^{\prime \prime} .5 \times 11^{\prime \prime} 8$ aperture). All the above values refer to the very central regions of the galaxy, corresponding to equivalent circular apertures (see Cappellari et al. 2006) from $\sim 1^{\prime \prime}$ to $1^{\prime \prime} .5$. We then normalized the values of the central velocity dispersion to a circular aperture of radius equal to the adopted $R_{\mathrm{e}}$, following the formula 


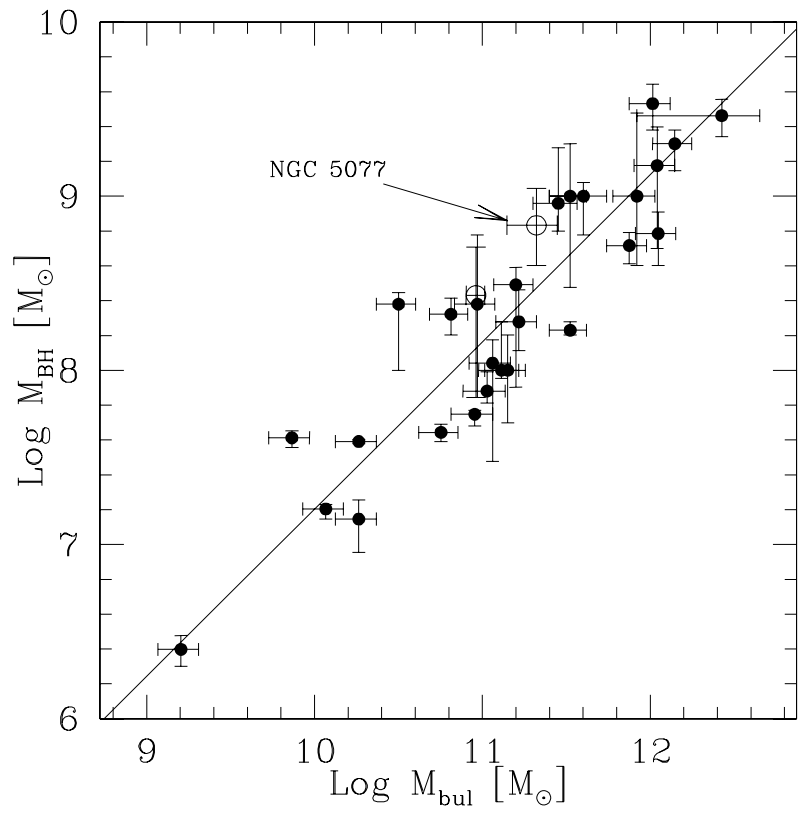

Fig. 12. $M_{\mathrm{BH}}$ vs. bulge mass from Marconi \& Hunt (2003) with the best fit obtained from a bisector linear regression analysis (solid line). The positions of NGC 5077 and NGC 3998 are shown by empty circles.

for aperture corrections by Cappellari et al. (2006). The average of these normalized values leads to $\sigma_{R_{\mathrm{e}}}=222 \pm 15 \mathrm{~km} \mathrm{~s}^{-1}$.

Using this value of $\sigma$ as an approximation for $\sigma_{\text {bul }}$, we obtained the value $M_{\text {bul }}=(2.1 \pm 0.7) \times 10^{11} M_{\odot}$ for the bulge mass. From the correlation of Marconi \& Hunt (2003), the expected $M_{\mathrm{BH}}$ for NGC 5077 is $3.0 \times 10^{8} M_{\odot}$, in agreement within a factor $\sim 2.3$, with our determination (see Fig. 12).

For the correlations between $\mathrm{BH}$ mass and central stellar velocity dispersion, one obtains $2.0 \times 10^{8} M_{\odot}$ for NGC 5077 adopting the form by Tremaine et al. (2002) (see Fig. 13, upper panel), a factor 3.4 lower than our estimate. We also compared our measurement with the expected value from the $M_{\mathrm{BH}}-\sigma$ relation derived by Ferrarese \& Ford $(2005)^{3}, M_{\mathrm{BH}}=5.4 \times 10^{8} M_{\odot}$, in full agreement with our estimate (see Fig. 13, bottom panel).

Conversely, the BH mass expected for NGC 5077 on the basis of its correlation with the Sérsic concentration index (from Trujillo et al. 2004) is a factor $5 \sim 6$ lower than our estimate when using the linear $M_{\mathrm{BH}}-$ Sérsic index correlation (Graham \& Driver 2007) and a factor 3 lower when using the quadratic form they proposed.

Marconi \& Hunt (2003) noticed that $M_{\mathrm{BH}}$ is separately correlated with both $\sigma$ and $R_{\mathrm{e}}$. This is shown by the weak correlation between the residuals of the $M_{\mathrm{BH}}-\sigma$ correlation with $R_{\mathrm{e}}$ reproduced here in Fig. 14 for the Tremaine at al. form ${ }^{4}$. Our determinations of the black hole mass in NGC 5077 and in NGC 3998 (Paper I) support this idea. Unlike NGC 3998, which has one of the lowest values of $R_{\mathrm{e}}$ among galaxies with measured $M_{\mathrm{BH}}(0.85 \mathrm{kpc})$ and shows a negative residual from the $M_{\mathrm{BH}}-\sigma$ correlation, NGC 5077 has an intermediate $R_{\mathrm{e}}$ value $(3.6 \mathrm{kpc})$ and show a small but positive residual (see Fig. 14). In the same sense, but with a larger positive residual, there is the result found by Capetti et al. (2005) for NGC 5252, a galaxy with quite a large effective radius $(9.7 \mathrm{kpc})$. This indicates the presence of a black

\footnotetext{
${ }^{3}$ We normalized the central velocity dispersion to an aperture of radius equal to $1 / 8$ of $R_{\mathrm{e}}$ and derived $\sigma_{\mathrm{R}_{\mathrm{e}} / 8}=255 \pm 17 \mathrm{~km} \mathrm{~s}^{-1}$.

4 A similar trend is obtained using the correlation in the Ferrarese et al. form.
}
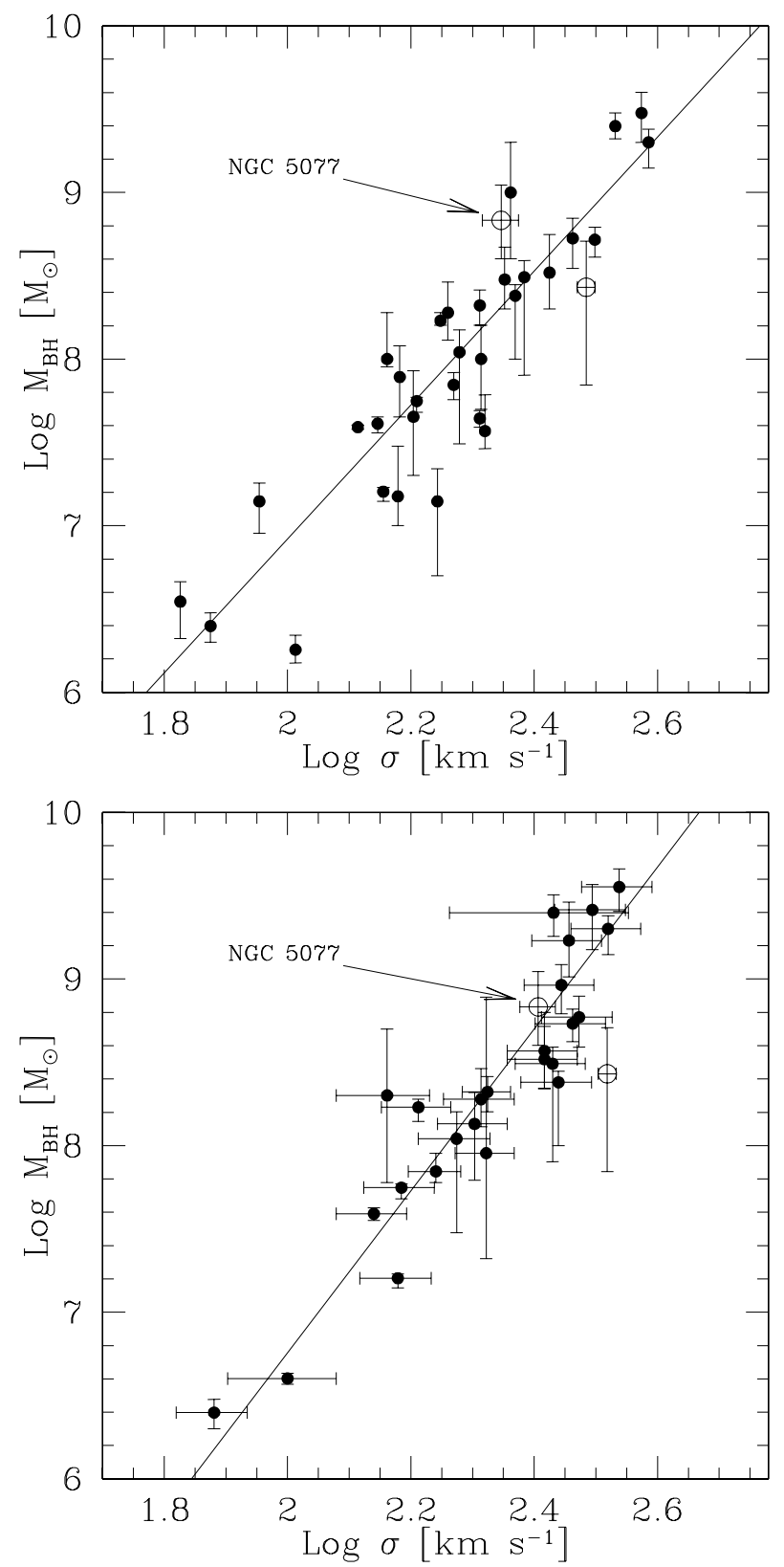

Fig. 13. $M_{\mathrm{BH}}$ vs. central stellar velocity dispersion from Tremaine et al. (2002) (upper panel) and from Ferrarese \& Ford (2005) (lower panel). Solid lines indicate the best fits for the two correlations. The positions of NGC 5077 and NGC 3998 are shown by empty circles.

hole's "fundamental plane" in the sense that a combination of at least $\sigma$ and $R_{\mathrm{e}}$ drives the correlations between $M_{\mathrm{BH}}$ and the bulge properties. The physical implications of these results will be discussed in a forthcoming paper, when new direct $\mathrm{BH}$ mass measurements are presented, in order to base our discussion on a statistically more significative sample of $M_{\mathrm{BH}}$ determinations.

\section{Summary and conclusions}

We have presented results from a gas kinematics study in the nucleus of three nearby LINER galaxies: IC 989, NGC 5077, and NGC 6500 using archival HST/STIS spectra. Only in the case of NGC 5077 does the nuclear velocity curves appear to be associated with gas in regular rotation. For IC 989 our results indicate an inner counter-rotating gas system, while for NGC 6500 the 


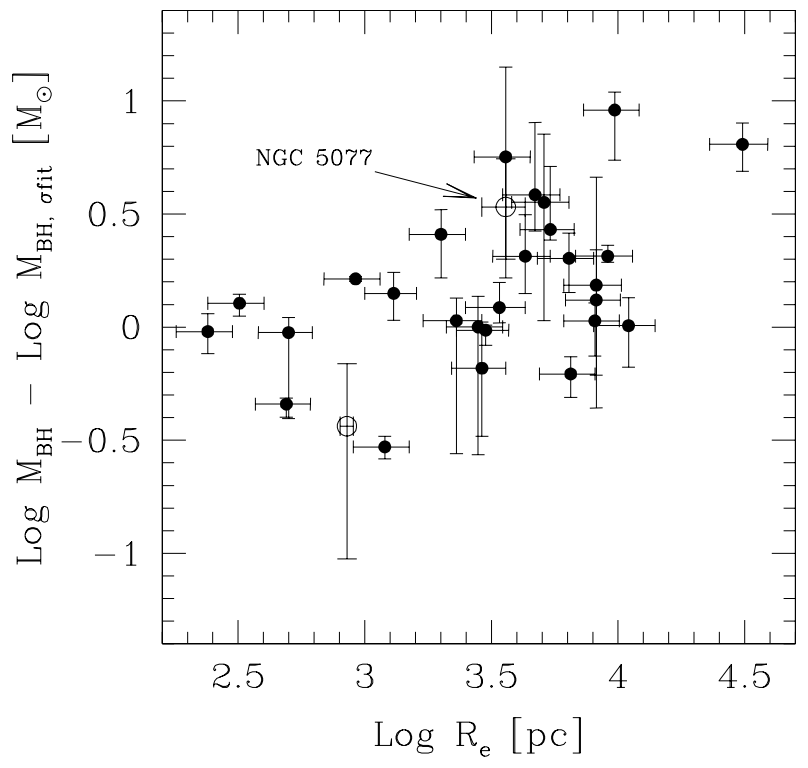

Fig. 14. Residuals from the $M_{\mathrm{BH}}$ vs. $\sigma$ correlation (in the Tremaine et al. form) reported against the bulge effective radius $R_{\mathrm{e}}$. The positions of NGC 5077 and NGC 3998 are indicated by empty circles.

complex trend in the velocity curves suggests a nuclear expanding bubble.

We used our modeling code to fit the observed [N II] $\lambda 6583$ surface brightness distribution and velocity curve of NGC 5077. The dynamics of the rotating gas can be accurately reproduced by motions in a thin disk when a compact dark mass of $M_{\mathrm{BH}}=6.8_{-2.8}^{+4.3} \times 10^{8} M_{\odot}$ is added to the stellar mass component. Furthermore, the black hole in NGC 5077 has a sphere of influence radius, $R_{\mathrm{sph}}=G M_{\mathrm{BH}} / \sigma_{\mathrm{star}}^{2}$, of $\sim 62 \mathrm{pc}(\simeq 0$ '! 34), wellresolved at the HST resolution $\left(2 R_{\mathrm{sph}} / R_{\mathrm{res}} \simeq 6.8\right)$.

For what concerns the connections of this $\mathrm{BH}$ mass estimate with the properties of the host galaxy, the $M_{\mathrm{BH}}$ value for NGC 5077 is in good agreement (within a factor of 2.3) with the $M_{\mathrm{BH}}-M_{\text {bul }}$ correlation between $\mathrm{BH}$ and host bulge mass. The black hole mass predicted by the $M_{\mathrm{BH}}-\sigma$ correlation is a factor of 3.4 lower than our measure, adopting the relation found by Tremaine et al. (2002) and in excellent agreement using the parameterization by Ferrarese \& Ford (2005).

This result, in conjunction with the previous results for NGC 3998 (Paper I), strengthens the possibility of a connection between the residuals from the $M_{\mathrm{BH}}-\sigma$ relation and the bulge effective radius. While NGC 3998, indeed, has one of the lowest values of $R_{\mathrm{e}}$ among galaxies with measured $M_{\mathrm{BH}}$ and shows a negative residual, NGC 5077 has a larger effective radius and shows a small positive residual. We also recently showed that the same result was found for the Seyfert galaxy NGC 5252: a larger effective radius corresponds in this galaxy to a still larger positive residual.

Apparently, only with a combination of at least $\sigma$ and $R_{\mathrm{e}}$ is it possible to account for the correlations between $M_{\mathrm{BH}}$ and other bulge properties, indicating the presence of a black hole's "fundamental plane". Clearly, the number of direct black-hole mass measurements must be further increased, together with precise determinations of $\sigma$ and $R_{\mathrm{e}}$, to test these conclusions on a stronger statistical basis. In a forthcoming paper we will present new $\mathrm{BH}$ mass determinations and discuss the physical implications of our results.

Acknowledgements. This publication makes use of the HyperLeda database, available at http://leda.univ-lyon1.fr, and of the NASA/IPAC Extragalactic Database (NED) operated by the Jet Propulsion Laboratory, California Institute of Technology, under contract with the National Aeronautics and Space Administration.

\section{References}

Barth, A. J., Ho, L. C., Filippenko, A. V., \& Sargent, W. L. W. 1998, ApJ, 496, 133

Barth, A. J., Filippenko, A. V., \& Moran, E. C. 1999, ApJ, 525, 673

Barth, A. J., Sarzi, M., Rix, H., et al. 2001, ApJ, 555, 685

Bender, R., Surma, P., Doebereiner, S., Moellenhoff, C., \& Madejsky, R. 1989, A\&A, 217, 35

Bertola, F., Bettoni, D., Danziger, J., et al. 1991, ApJ, 373, 369

Braatz, J. A., Wilson, A. S., \& Henkel, C. 1997, ApJS, 110, 321

Burstein, D., Davies, R. L., Dressler, A., et al. 1987, ApJS, 64, 601

Caon, N., Macchetto, D., \& Pastoriza, M. 2000, ApJS, 127, 39

Capetti, A., Marconi, A., Macchetto, D., \& Axon, D. 2005, A\&A, 431, 465 Cappellari, M., Verolme, E. K., van der Marel, R. P., et al. 2002, ApJ, 578, 787

Cappellari, M., Bacon, R., Bureau, M., et al. 2006, MNRAS, 366, 1126

Carollo, C. M., Danziger, I. J., \& Buson, L. 1993, MNRAS, 265, 553

Davies, R. L., Burstein, D., Dressler, A., et al. 1987, ApJS, 64, 581

De Francesco, G., Capetti, A., \& Marconi, A. 2006, A\&A, 460, 439

de Vaucouleurs, G., de Vaucouleurs, A., Corwin, Jr., H. G., et al. 1991, Third Reference Catalogue of Bright Galaxies, Vol. 1-3, XII (Berlin, Heidelberg, New York: Springer-Verlag)

Demoulin-Ulrich, M.-H., Butcher, H. R., \& Boksenberg, A. 1984, ApJ, 285, 527

Dressel, L. 2006, Instrument Science report STIS 2006-02

Ferrarese, L., \& Ford, H. 2005, Space Sci. Rev., 116, 523

Ferrarese, L., \& Merritt, D. 2000, ApJ, 539, L9

Ferrarese, L., Ford, H. C., \& Jaffe, W. 1996, ApJ, 470, 444

Gebhardt, K., Bender, R., Bower, G., et al. 2000, ApJ, 539, L13

Graham, A. W., \& Driver, S. P. 2007, ApJ, 655, 77

Graham, A. W., Erwin, P., Caon, N., \& Trujillo, I. 2001, ApJ, 563, L11

Haehnelt, M. G., \& Kauffmann, G. 2000, MNRAS, 318, L35

Harms, R. J., Ford, H. C., Tsvetanov, Z. I., et al. 1994, ApJ, 435, L35

Heckman, T. M. 1980, A\&A, 87, 152

Ho, L. C., Filippenko, A. V., \& Sargent, W. L. W. 1997a, ApJS, 112, 315

Ho, L. C., Filippenko, A. V., Sargent, W. L. W., \& Peng, C. Y. 1997b, ApJS, 112, 391

Kormendy, J., \& Richstone, D. 1995, ARA\&A, 33, 581

Krist, J., \& Hook, R. 1999, The Tiny Tim User's Guide (Baltimore:STDcI)

Macchetto, F., Marconi, A., Axon, D. J., et al. 1997, ApJ, 489, 579

Magorrian, J., Tremaine, S., Richstone, D., et al. 1998, AJ, 115, 2285

Maoz, D., Filippenko, A. V., Ho, L. C., et al. 1995, ApJ, 440, 91

Maraston, C. 1998, MNRAS, 300, 872

Marconi, A., \& Hunt, L. K. 2003, ApJ, 589, L21

Marconi, A., Axon, D. J., Capetti, A., et al. 2003, ApJ, 586, 868

Marconi, A., Pastorini, G., Pacini, F., et al. 2006, A\&A, 448, 921

Nagar, N. M., Falcke, H., Wilson, A. S., \& Ulvestad, J. S. 2002, A\&A, 392, 53

Poulain, P., \& Nieto, J.-L. 1994, A\&AS, 103, 573

Press, W. H., Teukolsky, S. A., Vetterling, W. T., \& Flannery, B. P. 1992, Numerical recipes in FORTRAN. The art of scientific computing (Cambridge: University Press, c1992, 2nd ed.)

Sánchez-Portal, M., Díaz, Á. I., Terlevich, E., \& Terlevich, R. 2004, MNRAS, 350,1087

Silk, J., \& Rees, M. J. 1998, A\&A, 331, L1

Tran, H. D., Tsvetanov, Z., Ford, H. C., et al. 2001, AJ, 121, 2928

Tremaine, S., Gebhardt, K., Bender, R., et al. 2002, ApJ, 574, 740

Trujillo, I., Erwin, P., Asensio Ramos, A., \& Graham, A. W. 2004, AJ, 127, 1917

van der Marel, R. P., \& van den Bosch, F. C. 1998, AJ, 116, 2220

Verdoes Kleijn, G. A., van der Marel, R. P., Carollo, C. M., \& de Zeeuw, P. T. 2000, AJ, 120, 1221

Verdoes Kleijn, G. A., van der Marel, R. P., de Zeeuw, P. T., Noel-Storr, J., \& Baum, S. A. 2002, AJ, 124, 2524 